\title{
Correspondance de La Beaumelle (1726-1773), éditée par Hubert Bost, Claude Lauriol et Hubert Angliviel de la Beaumelle
}

\section{Franco Piva}

\author{
(2) OpenEdition \\ Journals \\ Edizione digitale \\ URL: http://journals.openedition.org/studifrancesi/9565 \\ DOI: 10.4000/studifrancesi.9565 \\ ISSN: 2421-5856

\section{Editore} \\ Rosenberg \& Sellier

\section{Edizione cartacea} \\ Data di pubblicazione: 1 décembre 2007 \\ Paginazione: 666 \\ ISSN: 0039-2944

\section{Notizia bibliografica digitale} \\ Franco Piva, «Correspondance de La Beaumelle (1726-1773), éditée par Hubert Bost, Claude Lauriol et \\ Hubert Angliviel de la Beaumelle», Studi Francesi [Online], 153 (LI | III) | 2007, online dal 30 novembre \\ 2015, consultato il 13 janvier 2021. URL: http://journals.openedition.org/studifrancesi/9565; DOI: \\ https://doi.org/10.4000/studifrancesi.9565
}

Questo documento è stato generato automaticamente il 13 janvier 2021.

\section{cc) (†)}

Studi Francesi è distribuita con Licenza Creative Commons Attribuzione - Non commerciale - Non opere derivate 4.0 Internazionale. 


\title{
Correspondance de La Beaumelle (1726-1773), éditée par Hubert Bost, Claude Lauriol et Hubert Angliviel de la Beaumelle
}

\author{
Franco Piva
}

\section{NOTIZIA}

Correspondance de La Beaumelle (1726-1773), éditée par Hubert BOST, Claude LAURIOL et Hubert ANGLIVIEL DE LA BEAUMELLE, t. I (1729-1747), Oxford, Voltaire Foundation, 2005, pp. XXXIX+604.

1 Laurent Angliviel de La Beaumelle, noto soprattutto per le violente polemiche da lui ingaggiate con Voltaire, è un personaggio degno della massima attenzione per diversi aspetti: innanzitutto per le sue origini e per la sua formazione protestante che, se lo hanno in qualche modo marginalizzato, ne hanno però anche fatto un personaggio sui generis; non solo perché lo hanno costretto a campare, ancorché stentatamente, della sua penna, senza porsi al seguito di questo o quel protettore, o al riparo di questa o quella coterie, ma anche perché hanno costituito la peculiarità della sua intensa ancorché breve vita di homme de lettres, conducendolo attraverso l'Europa e ponendolo spesso en contrefaux delle posizioni ufficiali, fossero esse cattoliche, protestanti o illuministiche. All'inizio del movimento illuministico, al quale partecipa appieno, La Beaumelle ha infatti assunto posizioni spesso originali che lo hanno posto non di rado in rotta di collisione con molti dei suoi maggiori rappresentanti, salvo a trovare l'ammirazione di altri, come Montesquieu o La Condamine, che ne hanno apprezzato l'indipendenza di giudizio e la libertà di pensiero.

2 Dotato di un'acuta intelligenza e di una innata curiosità per ogni forma di sapere, lo troviamo presto intento a leggere tutto quanto di nuovo si pubblichi in Europa, e per 
ciò stesso in corrispondenza con molti degli uomini che al rinnovamento culturale di quel secolo hanno contribuito in vario modo. Sebbene Claude Lauriol abbia attirato l'attenzione degli studiosi su di lui già da diversi anni proprio sulla base di quanto la sua abbondante corrispondenza aveva consentito allo studioso di affermare, essa era rimasta finora quasi del tutto sconosciuta. Eppure si tratta di una delle maggiori corrispondenze del secolo. I curatori avvertono infatti che essa comprende oltre «3000 lettres, dont un tiers relève de la correspondance active, et deux tiers de la correspondance passive», mentre un altro migliaio sono «les lettres entre tiers qui parlent de lui ou mentionnent ses ouvrages», sicché i documenti relativi alla corrispondenza di La Beaumelle ammontano ad oltre 4000; la maggior parte di essi sono conservati negli archivi della famiglia Angliviel de La Beaumelle, che non solo li ha messi generosamente a disposizione dell'équipe che ha deciso di rendere finalmente pubbliche queste lettere, ma ha anche voluto partecipare all'impresa per il tramite di uno dei suoi discendenti.

3 L'operazione sarà per forza di cose lunga, ma a quanto si può arguire da questo primo volume, essa pare partita col piede giusto, e promette di porre presto a disposizione del pubblico, e più ancora forse degli studiosi del Settecento, una quantità straordinaria di informazioni che non potranno non riuscire di grande utilità, proprio per la particolare angolatura dalla quale gli avvenimenti di cui La Beaumelle è stato protagonista o testimone sono visti, vissuti e presentati. Il primo volume copre gli anni della formazione di La Beaumelle dapprima nel collegio cattolico dell'Enfance de Jésus ad Alès, nel quale il padre «nouveau converti» l'ha prudentemente posto, poi nella casa paterna a Valleraugue, dove lo sorprendiamo intento a divorare libri di ogni tipo, infine nell'Accademia protestante di Ginevra dove il giovane, ritornato alla fede primitiva della famiglia paterna, completa la sua formazione. Particolarmente importanti, a questo proposito, risultano le lettere che Laurent scambia con il fratello, anch'egli attento osservatore di quanto sta accadendo attorno a lui, anche se l'orizzonte del giovane Laurent si allarga presto ad altri ambiti ed entra in contatto con altri personaggi.

Ogni lettera è accompagnata da un'accurata annotazione che ci introduce dapprima nell'ambito della complessa famiglia degli Angliviels poi, via via, ci mette in rapporto con alcuni dei rappresentanti di quella comunità protestante, spesso condannata al silenzio quando non addirittura al «désert», con la quale La Beaumelle instaura rapporti sempre più stretti. La parte finale del volume comporta tutta una serie di documenti relativi a questi anni di formazione e si conclude con un utile indice dei nomi delle persone e delle opere citate nelle varie lettere. 